\title{
A COUPLED MULTI-PHYSICS MODEL FOR CREEP, SHRINKAGE AND FRACTURE OF EARLY-AGE CONCRETE
}

\author{
TOBIAS GASCH* ${ }^{*}$ ANDREAS SJÖLANDER ${ }^{\dagger}$, RICHARD MALM $^{\dagger \dagger}$ AND ANDERS \\ ANSELL ${ }^{\dagger \dagger}$
} \author{
Stockholm, Sweden \\ *e-mail: tobias.gasch@byv.kth.se \\ †e-mail: andreas.sjolander@byv.kth.se \\ ${ }^{\dagger \dagger}$ e-mail: richard.malm@byv.kth.se \\ †††e-mail: anders.ansell@byv.kth.se
}

KTH Royal Institute of Technology, Department of Civil and Architectural Engineering

Key words: Early-age, Shrinkage, Creep, Fracture, Multi-physics, Case study

\begin{abstract}
The behaviour of concrete at early-age is complex and involves several physical fields such as temperature, moisture and deformations. In this paper a hygro-thermo-chemo-mechanical model for the analysis of early-age concrete based on a combination of models from the literature is presented. The chemical model is based on the reaction degree concept, also used to define internal actions such as self-desiccation and ageing of mechanical properties. A mechanical model based on the Microprestress-Solidification theory for concrete creep is used, that in a simplified manner also considers concrete fracture. The model has been implemented in a numerical framework suitable for coupled multi-physics problems. It is here applied to a case study of an un-reinforced concrete tunnel plug made of a low-pH self-compacting concrete. Good agreement is generally obtained with measurements and hypotheses previously made on the behaviour of the plug are verified.
\end{abstract}

\section{INTRODUCTION}

The behaviour of concrete at early-age is a complex phenomenon for which the analysis necessarily has to include interaction of multiple physical fields such as temperature and moisture, to characterize its mechanical response. The perhaps most prominent phenomenon is the exothermic reaction of cement hydration, which for massive concrete structures may result in a significant temperature increase and thereby an increased risk for thermal cracking. Furthermore, the hydration reaction consumes water, leading to a moisture sink, self-desiccation, which causes autogenous shrinkage in the solid matrix. If the concrete is not cured properly this can lead to severe cracking, especially for concrete with low water/cement-ratios. Furthermore, the rate of hydration is highly influenced by the state of temperature and moisture, which affects the evolution of mechanical properties such as strength.

In this paper, a numerical model is presented that couples the evolution of temperature and moisture during the early-age of concrete with its mechanical behaviour. This model accounts for volumetric changes due to temperature and moisture variations as well as evolution of ma- 
terial properties during and after the initial hydration. Furthermore, the model also accounts for cracking through an isotropic damage model and creep by employing the MicroprestressSolidification (MPS) theory [1]. Similar coupled models for early-age concrete can be found in the literature, e.g. the model presented by Di Luzio and Cusatis [2]. In the second part of the paper, the model is applied to a case study of a large un-reinforced concrete structure.

\section{MODEL DESCRIPTION}

This section describes the overall structure of the mathematical model used in the current study. The proposed model combine models from the literature and for brevity only the most important aspects of and equations for the model are given.

\subsection{Hygro-thermo-chemical model}

Numerous models for analysing the earlyage behaviour of concrete have been presented in the literature, especially concerning the thermo-chemical aspects. One such model was presented by Ulm and Coussy [3], based on the thermodynamic framework of reactive porous media. This model was developed by Cervera et al. [4], that among others introduced a new concept for ageing. Using the same framework, Di Luzio and Cusatis [5] presented a model that in addition considered hygral aspects including moisture exchange with the environment, not dealt with previously. Furthermore, the model was also extended to better cover HighPerformance-Concrete by also including the silica fume $(\mathrm{SF})$ reaction and silicate polymerisation. The model model presented herein is largely based on the work presented in [3, 4, 5] and thus only the most important aspects and equations of the model are given below.

\subsubsection{Chemical reactions and ageing}

Although cement hydration in reality is a composition of chemical reactions, the literature often treats it as a single hydration process, since it has proved difficult to study the reactions. In the model developed by Ulm and Coussy [3], cement hydration is described by the so called hydration extent, or by the more practical measure: hydration degree $\alpha_{\mathrm{c}}$. This is defined as the ratio between the current hydration extent and its final theoretical value under ideal conditions. Thus, $\alpha_{\mathrm{c}}$ varies between 0 and 1 , but in reality ideal conditions never exist and a value of 1 is seldom reached [4, 5]. The evolution of $\alpha_{\mathrm{c}}$ can be described by equation (1) according to Di Luzio and Cusatis [5] and is driven by the normalized chemical affinity $A_{\mathrm{c}}\left(\alpha_{\mathrm{c}}\right)$.

$$
\frac{d \alpha_{\mathrm{c}}}{d t}=A_{\mathrm{c}}\left(\alpha_{\mathrm{c}}\right) \beta_{\mathrm{h}}(h) \exp \left(\frac{E_{\mathrm{c}}}{R T}\right)
$$

Cement hydration is a thermally activated reaction which is accounted for by the Arrheniusterm of the equation (last term), with $E_{\mathrm{c}}$ being the cement activation energy, $R$ the universal gas constant and the $T$ the temperature. Typically for concrete $E_{\mathrm{c}} / R=5000 \mathrm{~K}$ [4, 5]. Furthermore, an empirical function $\beta_{\mathrm{h}}(h)$ is also included to account for the decrease in reaction rate observed at low relative humidity. Here $\beta_{\mathrm{h}}(h)=\left[1+\left(a_{\mathrm{c}}-a_{\mathrm{c}} h\right)^{b}\right]^{-1}$ as proposed by Bažant et al. [6] with $a=5.5$ and $b=4$.

Since both the cement hydration and the SF reaction are largely driven by diffusion of water through the layers of reaction products formrf around the reaction compounds, Di Luzio and Cusatis [5] describe the SF reaction on a similar form as the cement hydration. The evolution of the SF reaction degree $\alpha_{\mathrm{s}}$ can therefore be described by equation (2), which as equation (1) is thermally activated and driven by a normalized chemical affinity $A_{\mathrm{s}}\left(\alpha_{\mathrm{s}}\right)$. For most cases $E_{\mathrm{s}} / R=9700 \mathrm{~K}$ can be assumed, where $E_{\mathrm{s}}$ is the activation energy of the SF reaction. In contrast to the cement hydration, it is not affected by the moisture state and thus unaffected by $h$.

$$
\frac{d \alpha_{\mathrm{s}}}{d t}=A_{\mathrm{s}}\left(\alpha_{\mathrm{s}}\right) \exp \left(\frac{E_{\mathrm{s}}}{R T}\right)
$$


The reaction associated with silicate polymerization that was included in the model by Di Luzio and Cusatis [5] has not been considered in the current work. However, since more than one reaction degree is used, it will be necessary to also introduce an overall reaction degree $\alpha$ for use in the ageing model. Following Di Luzio and Cusatis [2], $\alpha$ is given by equation (3) based on the amount of cement $c$ and silica fume $s$ and the heat developed by each respective reaction, where $\tilde{Q}_{\mathrm{c}}^{\infty}$ and $\tilde{Q}_{\mathrm{s}}^{\infty}$ are the total heat released for each complete reaction per unit mass.

$$
\alpha(t)=\frac{\alpha_{\mathrm{c}}(t) c \tilde{Q}_{\mathrm{c}}^{\infty}+\alpha_{\mathrm{s}}(t) s \tilde{Q}_{\mathrm{s}}^{\infty}}{c \tilde{Q}_{\mathrm{c}}^{\infty}+s \tilde{Q}_{\mathrm{s}}^{\infty}}
$$

The adopted ageing model follows the principles outlined by Cervera et al. [4], in which an ageing degree $\kappa_{\mathrm{f}}(\alpha)$ is postulated that describes the evolution of the compressive strength. Using the overall reaction degree as the driving variable, the aging degree is given as

$$
\frac{d \kappa_{\mathrm{f}}}{d t}=\lambda_{\mathrm{T}}(T) \lambda_{\alpha}(\alpha) \dot{\alpha}
$$

The function $\lambda_{\alpha}$ describes the growth of the ageing degree for different levels of reaction degree and can account for effects such as setting. Furthermore, the function $\lambda_{\mathrm{T}}$ accounts for the temperature influence on ageing, such that at lower curing temperatures the strength growth is higher. Based on the definition of $\kappa_{\mathrm{f}}$, the growth of different material properties can be defined, for example by following recommendations in design codes such as fib Model Code 2010 [7] as suggested by Cervera et al. [4]. For example, the tensile strength can be given as $f_{\mathrm{t}}=\kappa_{\mathrm{f}}^{2 / 3} f_{\mathrm{t}}^{\infty}$, where $f_{\mathrm{t}}^{\infty}$ is the tensile strength at $\kappa_{\mathrm{f}}=1$ and corresponds to strength growth under ideal isothermal conditions at a specified reference temperature. Similar expressions can be set up for other material properties such as the tensile fracture energy $G_{\mathrm{ft}}$.
Some parameters and variables are not described in detail above, for more information regarding these, the reader is referred to references [2, 3, 4]. The most notable variables are the normalized affinities $A_{\mathrm{c}}$ and $A_{\mathrm{s}}$ that ideally should be derived based on experimental data. However, an analytical expression was also suggested by Cervera et al. [4]. The asymptotic reaction degrees $\alpha_{\mathrm{c}}^{\infty}, \alpha_{\mathrm{s}}^{\infty}$ and $\alpha^{\infty}$ are needed in the analytical expressions for the affinities and also in some other parts of the model. These can be determined based on the composition of the concrete, see for example [2, 4].

\subsubsection{Heat transfer}

The heat transfer model follows the standard definition, where the heat flux is defined according to Fourier's law accompanied by the thermal energy balance equation. Since the cement and SF reactions are exothermic, an internal heat source $Q$ is included in the energy balance equation. This heat source is defined as $\dot{Q}=\dot{\alpha}_{\mathrm{c}} c \widetilde{Q}_{\mathrm{c}}^{\infty}+\dot{\alpha}_{\mathrm{c}} s \tilde{Q}_{\mathrm{s}}^{\infty}$ following Di Luzio and Cusatis [5].

\subsubsection{Moisture transfer}

Modelling moisture transport in concrete is a complex task where many models have been proposed in the literature; ranging from single to multi-phase models and models using different driving potentials such as moisture content $w$, relative humidity $h$ and vapour pressure $p_{\mathrm{v}}$. Regardless of which potential is chosen, common to most models is that the flow is governed by a diffusion-like equation. In this study, a single phase diffusion model is adopted with the relative humidity $h$ as the driving potential. This is a widely used type of model for moisture transport in concrete, although many more complex and accurate models can be found in the literature, e.g. the model of Gawin et al. [8]. The moisture mass balance used in this study is given by equation (5), where the total moisture content $w$ is the sum of the evaporable moisture content $w_{\mathrm{e}}$ and the non-evaporable mois- 
ture content $w_{\mathrm{n}}$ and is assumed to be in balance with the moisture flux $\mathbf{J}$ [5].

$$
\begin{aligned}
\frac{\partial w}{\partial t} & =\frac{\partial w_{\mathrm{e}}}{\partial t}+\frac{\partial w_{\mathrm{n}}}{\partial t}=-\nabla \cdot \mathbf{J} \\
& =\frac{\partial w_{\mathrm{e}}}{\partial h} \dot{h}+\frac{\partial w_{\mathrm{e}}}{\partial \alpha_{\mathrm{c}}} \dot{\alpha}_{\mathrm{c}}+\frac{\partial w_{\mathrm{e}}}{\partial \alpha_{\mathrm{s}}} \dot{\alpha}_{\mathrm{s}}+\dot{w}_{\mathrm{n}}
\end{aligned}
$$

As indicated by equation (5), $w_{\mathrm{e}}$ is assumed to be a function of the relative humidity $h$ and the previously introduced reaction degrees $\alpha_{\mathrm{c}}$ and $\alpha_{\mathrm{s}}$, which leads to an age-dependent sorption isotherm which is here given by equation (6). This definition splits $w_{\mathrm{e}}$ into moisture in gel pores (first term) and moisture in capillary pores (second term) and was first proposed by Norling Mjörnell [9]. The same sorption isotherm was used by Di Luzio and Cusatis [5] and for more details and definitions of all parameters in equation (6), the reader is referred to [5, 9].

$$
\begin{aligned}
w_{\mathrm{e}} & =G_{1}\left(\alpha_{\mathrm{c}}, \alpha_{\mathrm{s}}\right)\left(1-\frac{1}{\exp \left(\bar{g}\left(\alpha_{\mathrm{c}}\right) h\right)}\right) \\
& +K_{1}\left(\alpha_{\mathrm{c}}, \alpha_{\mathrm{s}}\right)\left(\exp \left(\bar{g}\left(\alpha_{\mathrm{c}}\right) h\right)-1\right)
\end{aligned}
$$

To complete the moisture balance in equation (5), definitions of the non-evaporable water $w_{\mathrm{n}}$ and the moisture flux $\mathbf{J}$ are needed. As discussed by Di Luzio and Cusatis [5], of the two studied reactions only the cement hydration consumes water. Thus $\dot{w}_{\mathrm{n}}=\kappa_{\mathrm{c}} c \dot{\alpha}_{\mathrm{c}}$, where $\kappa_{\mathrm{c}}=0.253$ can be assumed [5]. Lastly, as mentioned earlier, the moisture flux $\mathbf{J}$ is defined using a single driving potential $h$ with a humidity, age and temperature dependent transport coefficient, see equation (7). This definition of $\mathbf{J}$ was suggested by de Freitas Teixeira et al. [10] and the diffusivity $D_{\mathrm{w}}(h)$ proposed by Bažant and Najjar [11]. The temperature dependence is provided by the function $\Psi_{\mathrm{T}}(T)$ and the age (and humidity) dependence is given the moisture capacity $\partial w_{\mathrm{e}} / \partial h$.

$$
\mathbf{J}=-\Psi_{\mathrm{T}}(T) \frac{\partial w_{\mathrm{e}}}{\partial h} D_{\mathrm{w}}(h) \nabla h
$$

All partial derivatives of $w_{\mathrm{e}}$ used in equations (5) and (7) are calculated numerically.

\subsection{Mechanical model}

The mechanical model used is based on the standard momentum balance equation without inertial effects and on an assumption of small displacements and strains:

$$
\nabla \cdot \boldsymbol{\sigma}+\mathbf{b}=0 \text { and } \boldsymbol{\varepsilon}=\frac{1}{2}\left[\nabla \mathbf{u}+(\nabla \mathbf{u})^{\top}\right]
$$

The model accounts for effects such as moisture shrinkage $\varepsilon_{\mathrm{h}}$, thermal dilation $\varepsilon_{\mathrm{t}}$ as well as creep $\varepsilon_{\mathrm{v}}$ and $\varepsilon_{\mathrm{f}}$ as indicated by the additive strain decomposition in equation (9). The shrinkage and thermal strains are assumed directly proportional to the state variables $h$ and $T$ such that $\dot{\varepsilon}_{\mathrm{h}}=k_{\mathrm{h}} \dot{h} \mathbf{1}$ and $\dot{\varepsilon}_{\mathrm{t}}=k_{\mathrm{t}} \dot{t} \mathbf{1}$, where 1 is the second order unit tensor. Both expansion coefficients $k_{\mathrm{h}}$ and $k_{\mathrm{t}}$ are here assumed to be constant; however, this must be considered as a simplification, especially for the moisture induced shrinkage.

$$
\varepsilon=\varepsilon_{\mathrm{i}}+\varepsilon_{\mathrm{v}}+\varepsilon_{\mathrm{f}}+\varepsilon_{\mathrm{h}}+\varepsilon_{\mathrm{t}}
$$

The instantaneous strain tensor $\varepsilon_{\mathrm{i}}$ describes the age-independent part of the deformation. This strain measure is used in the constitutive law in equation (10), in which an isotropic scalar damage model is used to account for fracture:

$$
\boldsymbol{\sigma}=(1-\omega) \overline{\boldsymbol{\sigma}}=(1-\omega) \mathbb{D}_{\mathrm{i}}: \varepsilon_{\mathrm{i}}
$$

In equation $(10), \mathbb{D}_{i}$ is the elastic stiffness tensor, here given by the instantaneous modulus $E_{\mathrm{i}}=1 / q_{1}$, where $q_{1}$ is a material parameter of the MPS-theory [1], and the Possion's ratio $\nu$. This is assumed to be unaffected by damage and also equal for elastic and creep deformations. In this study, an isotropic scalar damage model have been chosen for concrete fracture 
due to its simplicity and robustness for large scale applications. The damage variable $\omega$ relates the effective stress $\bar{\sigma}$ to the nominal stress $\sigma$. Its evolution is given by an equivalent strain measure $\tilde{\varepsilon}$ in combination with a loading function and damage evolution laws. The choice of $\tilde{\varepsilon}$ depend on the application at hand, e.g. a definition according to Mazars and Pijaudier-Cabot [12] may be used, or if mainly tensile fracture is expected a Rankine type definition may be sufficient. The implemented damage model follows the framework outlined by Oliver et al. [13] and applies the crack band method [14] to regularize strain localization.

The creep model follows the MPS theory [1] and incorporates the later developments suggested by Bažant et al. [6], Jirásek and Havlásek [15] and Bažant et al. [16]. The rate of the solidifying viscoelastic strain $\dot{\varepsilon}_{\mathrm{v}}=\dot{\gamma}\left(t_{\mathrm{r}}\right) / v(\alpha)$, where $\gamma\left(t_{\mathrm{r}}\right)$ is a non-aging viscoelastic strain and $t_{\mathrm{r}}$ is a reduced time to account for general ambient conditions. The creep function used in the non-ageing viscoelastic model is defined according to [1], in which only a parameter $q_{2}$ is considered as a free model parameter. Furthermore, the creep function is approximated using the standard Dirichlet series expansion, which corresponds to a series of Kelvin-Voigt elements. The solidification function $v(\alpha)=1 /\left[\left(\alpha_{\infty} / \alpha\right)^{n_{\alpha}}\right]$ is here defined according to Di Luzio and Cusatis [2] so that it is merged with the used ageing model. The parameter $n_{\alpha}$ is a free model parameter and $\alpha_{\infty}$ is taken from the ageing model and is based on the concrete mixture. The rate of the viscous strain $\dot{\varepsilon}_{\mathrm{f}}=\psi_{\mathrm{r}}(h, T) \bar{\sigma} / \eta_{\mathrm{f}}(h, T)$ is governed by the effective stress. Furthermore, $\psi_{\mathrm{r}}$ is related to $t_{\mathrm{r}}$ and $\eta_{\mathrm{f}}$ is the microprestress viscosity introduced by Jirásek and Havlásek [15]. Introducing $\eta_{\mathrm{f}}$ eliminates the microprestress from the model formulation and the evolution of $\eta_{\mathrm{f}}$ is given by the following differential equation:

$$
\dot{\eta}_{\mathrm{f}}+\frac{1}{\mu_{\mathrm{s}} T_{0}}\left|T \frac{\dot{h}}{h}-\kappa_{\mathrm{T}}(T) \dot{T}\right|\left(\mu_{\mathrm{s}} \eta_{\mathrm{f}}\right)^{\tilde{p}}=\frac{\psi_{\mathrm{s}}}{q_{4}}
$$

The two main free model parameters in equation (11) is the fluidity $\mu_{\mathrm{s}}$ which replaces two parameters of the original model and parameter $q_{4}$ that depends on the concrete mixture. Variable $\kappa_{\mathrm{T}}$ was also introduced by Jirásek and Havlásek [15] to improve the model response under cyclic temperature conditions. A further improvement by Bažant et al. [16] is the parameter $\tilde{p}$ introduced to minimize the size effect. It was shown that $\tilde{p}=0.6$ minimizes the size effect, although this cause the original equations of the MPS-theory to lose their physical meaning. Lastly, the variable $\psi_{\mathrm{s}}$ is related to the evolution of the reduced microprestress time $t_{\mathrm{s}}$ that account for general ambient conditions and $T_{0}$ is a reference temperature. The above equations of the creep model are given for a onedimensional case. However, the extension to a three-dimensional case is straightforward, given isotropic behaviour and a constant Possion's ratio [1].

The above description only gives a brief overview of the mechanical model, but more information is given by Gasch et al. [17] and for the creep model in the original papers on the MPS-theory, e.g. [1, 6, 15, 16].

\section{IMPLEMENTATION}

The mathematical model outlined in section 2 has been implemented in the general FE code Comsol Multiphysics [18], using its equation based modelling feature. This feature allows input of arbitrary differential equations on either strong or weak form together with boundary and initial conditions. Furthermore, it is possible to couple equations describing different physics (typically PDEs) and those of internal variables (typically ODEs). The system of coupled PDEs and ODEs are discretized and assembled using shape functions individually specified for each dependent variable (state or internal). To avoid ill-conditioned system matrices, individual scaling factors are applied to each dependent variable in the discretized system, such that all variables are of a similar order of magnitude. In the current study the model is solved in series. First the hygro-thermo- 
chemo model (including $\eta_{\mathrm{f}}$ of the creep model) is solved followed by the mechanical model, with no coupling in the opposite direction considered. However, the implementation allows for the model to be solved in a fully-coupled system as well if a two-way coupling is necessary. This was shown by Gasch et al. [17] for a case where the permeability was made dependent on crack development.

\section{CASE STUDY}

A case study of a project carried out by the Swedish Nuclear Fuel and Waste Management Co (SKB) has been used to test the presented model. The project consists of a full-scale test casting of an un-reinforced concrete plug intended to seal deposition tunnels in a long-term nuclear fuel repository. The plug is an important part of the KBS-3V hard rock repository concept developed by SKB. A summary and more detailed description of the project is given by Malm [19] and Grahm et al. [20]. Apart from the actual test casting, the project also included material development and testing, where a low-pH self-compacting concrete (SCC) was developed. The motivation of using concrete with low-pH is to protect the bentonite backfill which loses its chemical integrity if the $\mathrm{pH}>11$, where concrete normally has a $\mathrm{pH}>12.5$. The material development and mix design of the low-pH SCC is described in detail by Vogt et al. [21] with the recipe of the concrete used in the test casting given in Tab. 1 .

Table 1: Composition of the low-pH SCC [21].

\begin{tabular}{lc}
\hline & $\mathrm{kg} / \mathrm{m}^{3}$ \\
\hline CEM I 42.5 MH/SR/LA & 120 \\
Silica fume & 80 \\
Water & 165 \\
Limestone filler & 369 \\
Sand 0-8 mm & 1037 \\
Gravel 8-16 mm & 538 \\
Glenium 51 & 6.38 \\
\hline
\end{tabular}

\subsection{Material testing}

The material testing performed by Vogt et al. [21] has been used to calibrate the free parameters of the presented model. The most important tests and results are described in the following, but due to a lack of space all performed simulations are not presented. One of the most characteristic features of the low-pH SCC is its high early-age shrinkage, and therefore several tests were performed to quantify this property. In Fig. 1, test results obtained using a dilatometer is shown with the corresponding prediction by the calibrated hygro-thermochemical model. Observed is the rapid initial shrinkage followed by a plateau before the shrinkage again accelerates. In context of the model, the first regime is explained by the cement hydration, which due to low cement content reaches its asymptotic reaction degree after only a few days. It then takes approximately two weeks before the silica fume reaction starts to accelerate and the shrinkage continues.

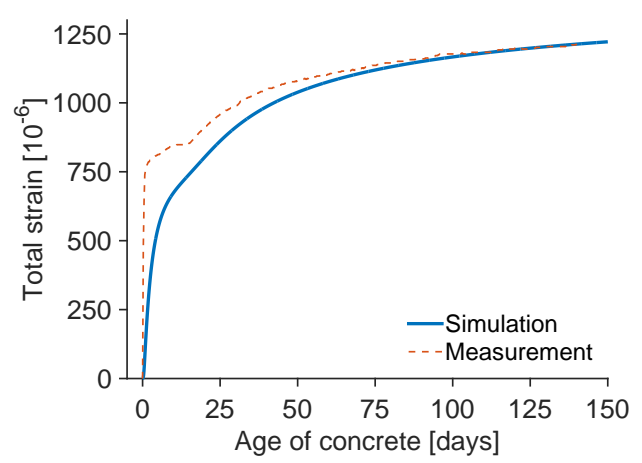

Figure 1: Early-age shrinkage as measured with a dilatometer [21].

The evolution of the compressive strength under isothermal conditions $\left(20{ }^{\circ} \mathrm{C}\right)$ is shown in Fig. 2. Since both the chemical shrinkage and the evolution of strength are driven by the reaction rates there is a trade off in the model calibration given the available measurements. If the strength growth is to be captured accurately, the rate of the initial chemical shrinkage is underestimated and vice versa. It should, however, be mentioned that the two tests in Figs. 1 and 2 were performed at different laboratories and 
that there are some uncertainties in the boundary conditions. This could explain the differences in the observed rate of the reaction from the measurements.

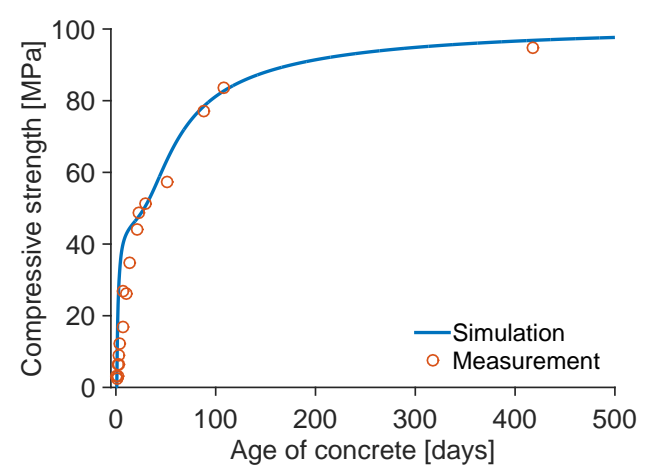

Figure 2: Evolution of compressive strength as predicted by the model compared to measurements [21].

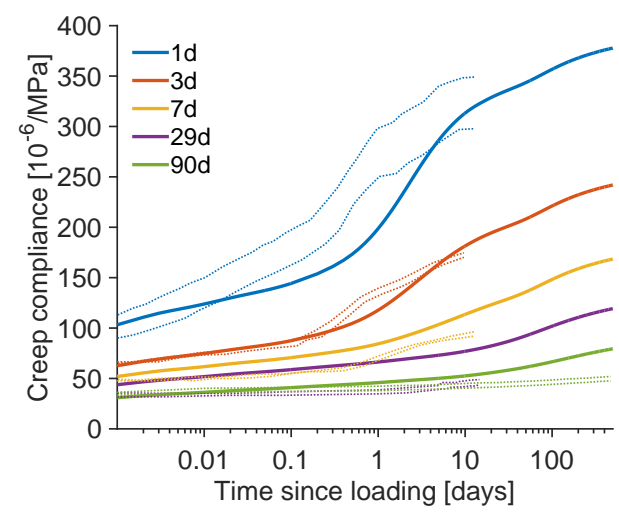

Figure 3: Creep compliance measured at different ages of loading. Solid lines show simulation results while dashed lines show measurements.

The last material test presented in this paper is on basic creep of the low-pH SCC, as reported for loading ages between 1 to 29 days in [21] and also for 90 days in [20]. The compliance obtained from the experimental results and the corresponding analysis results are shown in Fig. 3, for five ages of loading. Note that for the four earliest ages, the measurements only lasted for 10 days, whereas for the test started at 90 days the measurements are planned to continue for three years. It proved difficult to get a perfect fit for all loading ages and the current set of model parameters slightly overestimates creep at ages between 7-30 days, but the fit is otherwise good. What should be mentioned is that the early-age creep is highly influenced by the self-desiccation where consequently drying creep plays an important role. Thus, even though no direct tests on drying creep were performed, the tests on early-age basic creep provide some indication to the sensitivity and magnitude of the low-pH SCC to drying creep.

Table 2: Free model parameters used in the calibrated hygro-thermo-chemical model.

\begin{tabular}{lcrl}
\hline Cement affinity & $A_{\mathrm{c} 1}$ & $1.8 \cdot 10^{7}$ & $1 / \mathrm{h}$ \\
$A_{\mathrm{c}}\left(\alpha_{\mathrm{c}}\right)$, see [4, 5] & $A_{\mathrm{c} 2}$ & $10^{-5}$ & - \\
& $\eta_{\mathrm{c}}$ & 4 & - \\
Cement latent heat & $\tilde{Q}_{\mathrm{c}}^{\infty}$ & 117 & $\mathrm{~kJ} / \mathrm{kg}$ \\
\hline SF affinity & $A_{\mathrm{s} 1}$ & $1.8 \cdot 10^{12}$ & $1 / \mathrm{h}$ \\
$A_{\mathrm{s}}\left(\alpha_{\mathrm{s}}\right)$, see [4, 5] & $A_{\mathrm{s} 2}$ & $10^{-3}$ & - \\
& $\eta_{\mathrm{s}}$ & 4 & - \\
SF latent heat & $\tilde{Q}_{\mathrm{c}}^{\infty}$ & 780 & $\mathrm{~kJ} / \mathrm{kg}$ \\
\hline Conductivity & $\lambda_{\mathrm{T}}$ & $1.3^{*}$ & $\mathrm{~W} /(\mathrm{m} \cdot \mathrm{K})$ \\
Specific heat & $C_{\mathrm{p}}$ & $880^{*}$ & $\mathrm{~J} /(\mathrm{kg} \cdot \mathrm{K})$ \\
\hline Sorption isotherm & $g_{1}$ & 1.15 & - \\
$w_{e}$, see [5] & $k_{\mathrm{vg}}^{\mathrm{c}}$ & 0.1 & - \\
& $k_{\mathrm{vg}}^{\mathrm{s}}$ & 1.2 & - \\
\hline Diffusivity & $D_{1}$ & $2 \mathrm{e}-7^{*}$ & $\mathrm{~m} / \mathrm{s}$ \\
$D_{\mathrm{w}}(h)$, see [11] & $a_{0}$ & $0.05^{* *}$ & - \\
& $h_{\mathrm{d}}$ & $0.8^{* *}$ & - \\
& $n_{\mathrm{d}}$ & $15^{* *}$ & - \\
\hline Aging parameter & $A_{\mathrm{f}}$ & 2.25 & - \\
Temperature effect & $n_{\mathrm{T}}$ & 0.3 & - \\
Initial set & $\alpha_{0}$ & 0.125 & - \\
\hline
\end{tabular}

* Assumed value
** Value taken from fib Model Code 2010 [7]

The free parameters of the model calibrated to the low-pH SCC are summarized in Tabs. 2 and 3 . Some additional model parameters such as $\alpha_{\mathrm{c}}^{\infty}, \alpha_{\mathrm{s}}^{\infty}$ and $\alpha^{\infty}$ are needed, and can, as previously mentioned, be calculated on basis of the used concrete mixture, see Tab. 1. However, it should be pointed out that the high content of limestone filler in the low-pH SCC must be considered, although it is not implicitly accounted for in the mathematical model. Here, this is done by interpreting the w/c ratio as water over the combined cement plus filler content in order 
to get a reasonable amount of water consumption to account for the high shrinkage. The latent heat of the reaction $\widetilde{Q}_{\mathrm{c}}^{\infty}$ is reduced accordingly, so that the total heat produced only reflects the actual cement content. Using such an adjustment, the temperature development of the model fits experimental results during the initial hours when only cement hydration is active. It also give reasonable results for the early-age shrinkage as reported in Fig. 1 .

Table 3: Free model parameters used in the calibrated mechanical model.

\begin{tabular}{lcrl}
\hline Expansion coeff. & $k_{\mathrm{h}}$ & 0.0052 & - \\
& $k_{\mathrm{T}}$ & $1.0 \cdot 10^{-5}$ & $1 / \mathrm{K}$ \\
\hline Comp. strength & $f_{\mathrm{c}}^{\infty}$ & 95 & $\mathrm{MPa}$ \\
Tensile strength & $f_{\mathrm{t}}^{\infty}$ & 4.7 & $\mathrm{MPa}$ \\
Fracture energy & $G_{\mathrm{ft}}^{\infty}$ & $200^{* *}$ & $\mathrm{~J} / \mathrm{m}^{2}$ \\
\hline MPS parameters & $q_{1}$ & $5 \cdot 10^{-6}$ & $1 / \mathrm{MPa}$ \\
& $q_{2}$ & $6.2 \cdot 10^{-5}$ & $1 / \mathrm{MPa}$ \\
& $q_{4}$ & $5.0 \cdot 10^{-6}$ & $1 / \mathrm{MPa}$ \\
Solidification & $n_{\alpha}$ & 2.5 & - \\
\hline Fluidity & $\mu_{\mathrm{s}}$ & $2.6 \cdot 10^{-11}$ & $1 /(\mathrm{MPa} \cdot \mathrm{d})$ \\
Thermal creep & $\kappa_{\mathrm{Tm}}$ & $0.2^{*}$ & - \\
$\kappa_{\mathrm{T}}$, see [15] & $\kappa_{\mathrm{Tc}}$ & $0.02^{*}$ & - \\
\hline
\end{tabular}

\subsection{Structural test}

The sealing plug planned to be used in the KBS-3V nuclear repository is a dome construction, with an un-reinforced concrete plug cast inside a rock slot. A schematic layout of the plug is shown in Fig. 4 where it is assumed to have a rotational symmetry along the tunnel direction. Included in the figure is also a number of cooling pipes, the use of which is twofold. Primarily they are used to control the temperature rise during casting. The secondary function is to create a temperature pre-stressing of the dome, where the idea is to cool the concrete dome so that it releases from the rock followed by a contact grouting in the interface zone. Before construction of the concrete plug, the deposition is filled with bentonite clay, which serves as the main barrier in the planned repository. Hence, the concrete plug is only intended as a temporary seal during operation of the reposi- tory. A section of approximately $1 \mathrm{~m}$ bentonite backfill is included in the full-scale test. As the bentonite homogenizes with water, it can swell several orders of magnitude, however ,this can take several years before. To simulate this in the full-scale test, a water pressure of up to 4 MPa was created in the backfill. However, this part of the test program will not be analyzed in this study which focus on the first 200 days after casting.

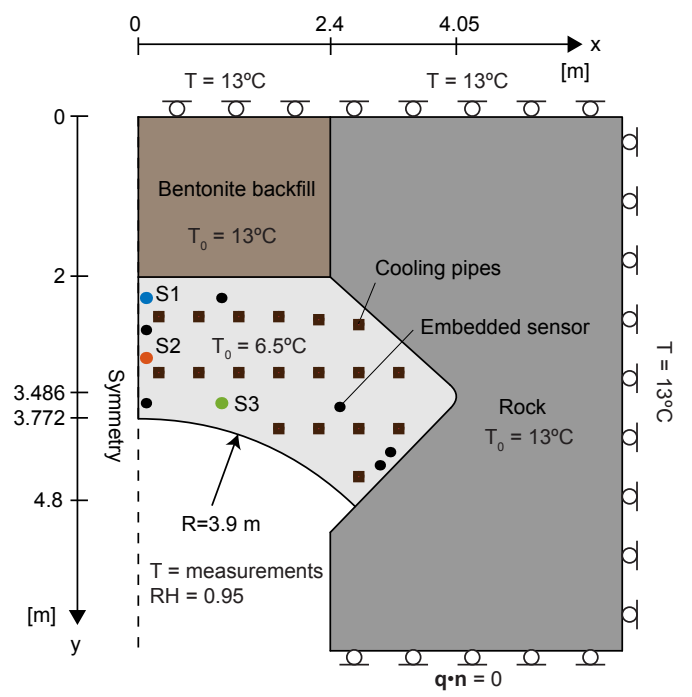

Figure 4: Geometry of the dome plug, including layout of cooling pipes and embedded sensors. The figure also shows the most important boundary and initial conditions.

The full-scale structural test of the dome plug was conducted at the Äspö Hard Rock Laboratory in Sweden, where casting occurred in March 2013. A photo of the downstream domed surface of the plug after casting is shown in Fig. 5. Before casting, 27 combined temperature and strain sensors were installed together with some additional sensors, e.g. ambient temperature and displacement gauges. A schematic layout of the embedded sensors is included in Fig. 4. These are installed in three radial planes with eight sensors in each plane and measure radial strains, see [20]. Results will later be shown for three of these locations, where for further reference the blue sensors is referred to as $\mathrm{S} 1$, the red as $\mathrm{S} 2$ and the green as $\mathrm{S} 3$. 


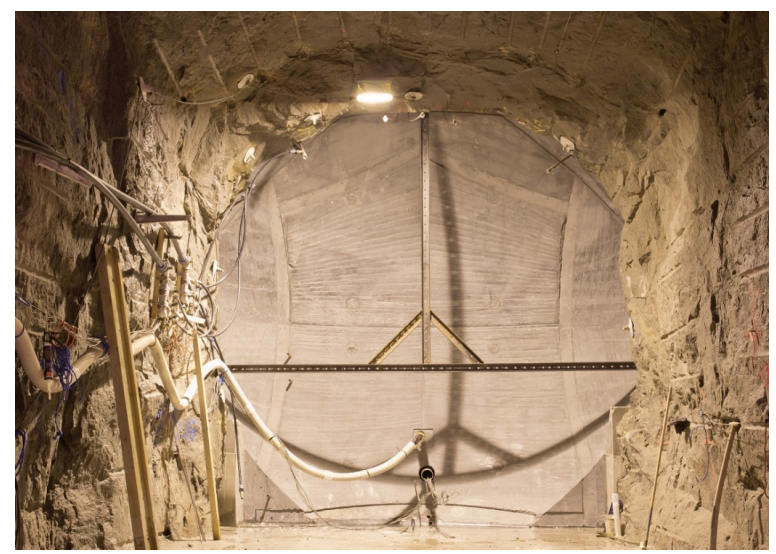

Figure 5: Dome plug after casting, photo from Grahm et al. [20].

The numerical model set up assumes axial symmetry, where the axis of symmetry is in the direction of the tunnel. The geometry and the main boundary conditions are included in Fig. 4. Moisture transport is only modelled in the concrete part of the geometry, where boundary conditions towards the rock and the backfill are set as $h=1$. The mesh is constructed with triangular elements with a higher element density for the concrete volume where all physical variables are discretized using linear Lagrange shape functions. The interface between concrete and rock is modelled using a cohesive zone model (CZM) that only consider pure mode 1 fracture. The properties of the CZM are set to depend on the fracture properties of the concrete. But to direct a fracture to the interface zone, its bond strength is set to $75 \%$ of the tensile concrete strength. However, a localized fracture in the interface is expected to be very brittle, so to artificially stabilize the model and improve convergence, the fracture energy of the CZM is increased to obtain a more ductile behaviour. Both the formwork and the backfill are in the displacement part of the analysis modelled using spring elements connected to the ground, with zero stiffness in tension. The effect of the formwork is removed after three weeks [20]. The cooling pipes (Fig. 4) are in the heat transfer described as point sources (i.e. lines in 3D) with a prescribed temperature. Figure 6 shows the temperature in the cooling pipes as well as the measured air temperature.

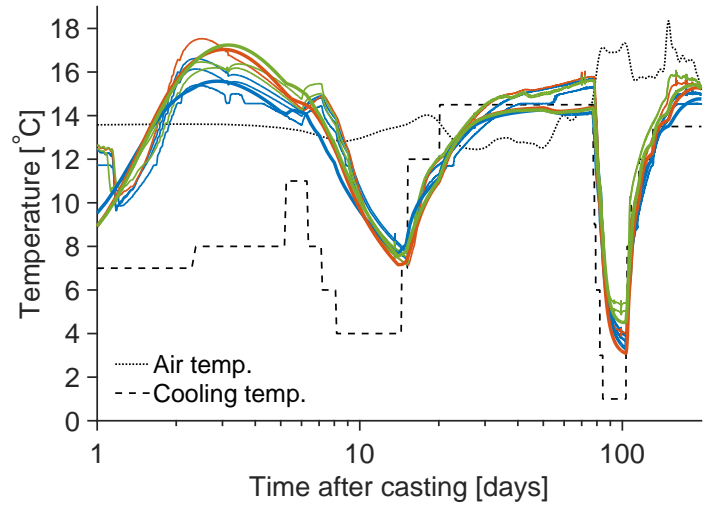

Figure 6: Temperature development at three sensor locations (S1, S2 and S3) with up to three sensor readings at each location, see Fig. 4 Bold lines represent simulations and thin lines correspond to measurements

Temperature readings at three selected sensor locations are shown in Fig. 6 together with the corresponding analysis results, with colours according to Fig. 4. The overall agreement is very good and the analysis and measurements follows the same trends; largely governed by the prescribed temperature of the cooling pipes. As expected, it is difficult to capture the exact behaviour during the first days since the continuous pour of the actual casting took 10 hours [20], while in the analysis the concrete is poured instantly. However, the maximum temperature reached during cement hydration is captured accurately by the model but the peak is generally delayed if compared to the measurements. The temperature field obtained in the analysis is shown for different times in Fig. 7

The strain history of the dome plug proved to be sensitive to the behaviour of the interface zone in combination with the early-age creep properties of the low-pH SCC. In fact, during evaluation of the test results, no conclusive statement could be made on whether the dome had released from the rock as intended [20]. Comparing the measured strain levels which have a magnitude of approximately 100 microstrain with the measured free early-age shrinkage (Fig. 11) indicates that either the plug is constrained so that the majority of the shrinkage strain is relaxed by the high early creep, or the free shrinkage smaller than expected. 


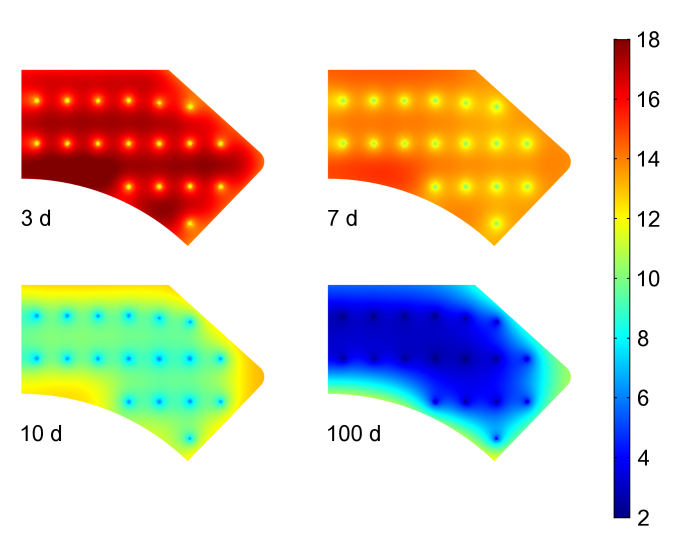

Figure 7: Temperature field in ${ }^{\circ} \mathrm{C}$ after 3, 7, 10 and 100 days.

For sensor S1 (located towards the backfill) the strain history is shown Fig. 8a, where the zero level for both measured and calculated strains are set at peak temperatures. The thermal component of the strain history has been omitted in Fig. 8a, so that the effect of the concrete creep is visualized. Studying different levels of interface (and concrete) strengths, it is evident that there are high restraint stresses in the concrete throughout the analyzed period. For simulations where there is significant debonding, these disappear. Thus it can be concluded that the most likely scenario is that the dome, at least partially, never released from the rock, although the strengths required for such scenario in the simulations by far exceeds the measured values of the low-pH SCC.

However, during the first 20-25 days the model is not able to predict the measurements with the desired accuracy. This is even more evident if the results from sensor S2 are studied in Fig. 8b. The simulations clearly overestimate the amount of creep during this period, or there may be factors not accounted for. One possibility could be that the bond at the interface has not developed fully during this period. On the other hand the low-pH SCC exhibits a large amount of early shrinkage which would make it difficult to later form a perfect bond over the entire circumference. Also, the model assumes that both expansion coefficients $k_{\mathrm{h}}$ and $k_{\mathrm{t}}$ are constant, a simplification that might explain the observed deviation. For example, $k_{\mathrm{t}}$ varies with the moisture content and also with the reaction degree. The same is true for $k_{\mathrm{h}}$. A lower $k_{\mathrm{t}}$ during the first days could explain the lower amount of observed creep strains. Another possibility is that there is a difference between the concrete mixture used in the laboratory testing (section 4.1) and that used in the structural casting caused by, e.g. the use of retarders to alleviate transport from the factory. This could for example affect the observed shrinkage, but it is unlikely that it would cause the concrete to exhibit lower compliance at early ages than later on, as indicated by the measurements.

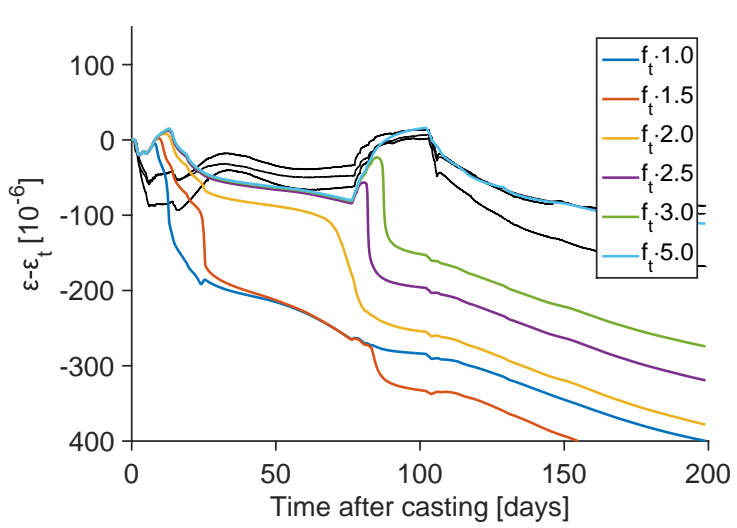

(a)

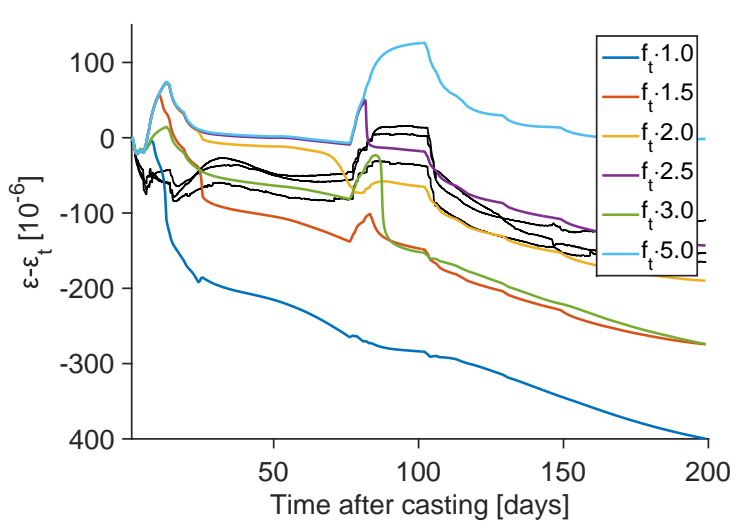

(b)

Figure 8: Strain history of total strain $\varepsilon$ minus thermal strain $\varepsilon_{\mathrm{t}}$ at sensor location (a) S1 and (b) S2, see Fig. 4 Black curves show measurements.

Lastly, the simulated gap at four locations along the concrete-rock interface for different values of strength is shown in Fig. 9. It can be seen that it is only the case with the low- 
est strength that results in a complete release of the dome, while for the highest strength no gap is observed. Although no measurements are included in Fig. 9, Grahm et al. [20] report measured gaps lower than $0.1 \mathrm{~mm}$ from the full-scale test which is in good agreement with a simulation using the highest strength value.

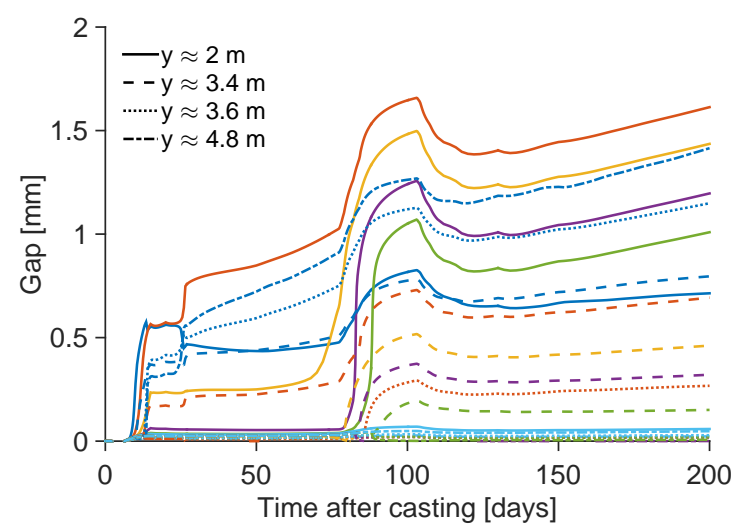

Figure 9: Simulated gap in the concrete-rock interface at different y-axis coordinates (Fig. 4) for different strength values (colour according to Fig. 8).

\section{CONCLUSIONS}

In this study, a model for the hygro-thermochemo-mechanical behaviour of early-age concrete is presented, based on a combination of different models from the literature. It was discussed how to implement the proposed model in a flexible numerical framework especially suitable for coupled multi-physics problems. Although the model treats the chemical behaviour of concrete using two reaction (cement and silica fume), the presented case study highlighted a shortcoming for the low-pH SCC used which contains a high amount of limestone filler. To accurately describe such concretes types, the hygro-thermo-checmical part of the model should be extended to also cover chemically active fillers. Still, it was shown that the model could describe the overall behaviour of the studied concrete plug. However, it proved difficult to completely characterize the behaviour of the concrete rock interface, where the measurements indicate a partial de-bonding. This part of the analysis has a significant influence on the results obtained.

\section{ACKNOWLEDGEMENTS}

The research presented was carried out as a part of "Swedish Hydropower Centre -SVC". SVC has been established by the Swedish Energy Agency, Energiforsk and Svenska Kraftnät together with Luleå University of Technology, KTH Royal Institute of Technology, Chalmers University of Technology and Uppsala University. www.svc.nu.

All experimental works were carried out and financed within the research program for a future long-term nuclear fuel repository of the Swedish Nuclear Fuel and Waste Management Co (SKB).

\section{References}

[1] Z. P. Bažant, A. Hauggaard, S. Baweja, and F. Ulm. Microprestress-Solidification Theory for Concrete Creep.I: Aging and Drying Effects. Journal of Engineering Mechanics, 123(11):1188-1194, 1997.

[2] G. Di Luzio and G. Cusatis. Solidification-microprestress-microplane (SMM) theory for concrete at early age: Theory, validation and application. International Journal of Solids and Structures, 50(6):957-975, 2013.

[3] F.-J Ulm and O. Coussy. Strength Growth as Chemo-Plastic Hardening in Early Age Concrete. Journal of Engineering Mechanics, 122(12):1123-1132, 1996.

[4] M. Cervera, J. Oliver, and T. Prato. Thermo-Chemo-Mechanical Model for Concrete. I: Hydration and Aging. Journal of Engineering Mechanics, 125(9):10181027.

[5] G. Di Luzio and G. Cusatis. Hygrothermo-chemical modeling of high performance concrete. I: Theory. Cement and Concrete Composites, 31(5):301308, 2009.

[6] Z. P. Bažant, G. Cusatis, and L. Cedolin. Temperature Effect on Concrete Creep Modeled by Microprestress-Solidification 
Theory. Journal of Engineering Mechanics, 130(6):691-699, 2004.

[7] fib. fib Model Code for Concrete Structures 2010. Wiley-VCH Verlag GmbH \& Co. KGaA, 2013. ISBN 978-3433030615.

[8] D. Gawin, F. Pesavento, and B. A. Schrefler. Hygro-thermo-chemo-mechanical modelling of concrete at early ages and beyond. Part I: hydration and hygrothermal phenomena. International Journal for Numerical Methods in Engineering, 67(3):299-331, 2006.

[9] K. Norling Mjörnell. Moisture conditions in high performance concrete: mathematical modelling and measurements. $\mathrm{PhD}$ Thesis, Chalmers University of Technology, 1997.

[10] J. A. de Freitas Teixeira, P. T. Cuong, and R. Faria. Modeling of cement hydration in high performance concrete structures with hybrid finite elements. International Journal for Numerical Methods in Engineering, 103(5):364-390, 2015.

[11] Z. P. Bažant and L. J. Najjar. Nonlinear water diffusion in nonsaturated concrete. Matriaux et Construction, 5(1):320, 1972.

[12] J. Mazars and G. Pijaudier-Cabot. Continuum Damage TheoryApplication to Concrete. Journal of Engineering Mechanics, 115(2):345-365, 1989.

[13] J. Oliver, M. Cervera, S Oller, and J. Lubliner. Isotropic damage models and smeared crack analysis of concrete. In N. Bicanic and H. A. Mang, editors, Computer Adided Analysis and Design of Concrete Strucutres, pages 945-957, Swansea, U.K., 1990. Pineridge Press.
[14] Z. P. Bažant and B. H. Oh. Crack band theory for fracture of concrete. Matriaux et Construction, 16(3):155-177, 1983. doi: 10.1007/BF02486267.

[15] M. Jirásek and P. Havlásek. Microprestress-solidification theory of concrete creep: Reformulation and improvement. Cement and Concrete Research, 60(0):51-62, 2014.

[16] Z. P. Bažant, P. Havlásek, and M. Jirásek. Microprestress-solidification theory on drying creep. In Computational Modelling of Concrete Structures, pages 749-758. CRC Press, 2014.

[17] T. Gasch, R. Malm, and A Ansell. A coupled hygro-thermo-mechanical model for concrete subjected to variable environmental conditions. International Journal of Solids and Structures, 2016. To be published.

[18] COMSOL. COMSOL Multiphysics ver 5.2 Documentation. COMSOL AB, Stockholm, Sweden, 2015.

[19] R. Malm. Low-pH concrete plug for sealing the KBS-3V deposition tunnels. SKB R-11-04, Swedish Nuclear Fuel and Waste Management Co (SKB), 2012.

[20] P. Grahm, R. Malm, and D. Eriksson. System design and full-scale testing of the Dome Plug for KBS-3V deposition tunnels. SKB TR-14-23, Swedish Nuclear Fuel and Waste Management Co (SKB), 2014.

[21] C. Vogt, B. Lagerblad, F. Baldy, and J.-E. Jonasson. Low $\mathrm{pH}$ self compacting concrete for deposition tunnel plugs. SKB R09-07, Swedish Nuclear Fuel and Waste Management Co (SKB), 2009. 